\title{
Electronic acute medical take - small measures to improve transparency, management, flow and efficiency; a model for the future hospital
}

\author{
Authors: Danielle Lux, ${ }^{A_{*}}$ Rachel Darnell, ${ }^{A}$ Karen $\mathrm{Kee}^{\mathrm{A}}$ and Craig Burke ${ }^{\mathrm{B}}$
}

\begin{abstract}
Introduction
Increasing demands on the emergency department (ED) and acute medicine over the past decade have tested quality and management, unmasking long-accepted inefficient and archaic systems. More robust processes are needed to identify workflow and resource pressures in a more timely manner. Starting at the front door, the referral system between the ED and medicine is obscure and rife with inherent delays and consequent compromised patient care. A lack of transparency confounds performance and collaboration. The medical registrar, often the most experienced doctor on site, is hindered from clinical utility in fielding bleep referrals. Strategies to improve the acute medical take, including optimising the utility and efficiency of the medical registrar, are needed. With the increasing importance of leadership and management as core skills, medical registrars need an opportunity to develop an oversight role, as stated in the JRCPTB quality criteria, while also benefiting their juniors in accessibility for educational purposes.
\end{abstract}

\section{Materials and methods}

Process-mapping was used to evaluate the current system of acute referral to medicine. Shadowing of junior doctor work behaviours over shift patterns, in addition to staff surveys, highlighted various barriers to productivity, clinical effectiveness and flow. Accessibility, reliability and the static nature of the current Excel spreadsheet system utilised were explored. We collaborated with Patienteer, a task management software tool, to develop an acute medical take list generated from extracted live data from Cerner. Baseline measures were taken for key performance indicators (KPIs; length of stay), time to clerking, time to senior decision-making and time to ED departure. An electronic tool was developed, extracting information from Cerner and using it to automatically generate a 'live' list of referred patients, with escalation by care or flow, ability to generate a task list, including senior review and increased transparency between not just the ED and medicine but also with the site management team with particular regard to bed management. A pilot study was performed looking at multiple KPIs, flow, educational metrics and satisfaction.

\section{Results}

The live electronic list demonstrated reduced time to senior decision-making (namely, decision to admit or discharge), reduced length of stay in department (reduced 12-hour breaches), improved flow through ED (results pending) and improved staff satisfaction. Unwell patients were identified and seen earlier. Work volume was more consistent, with less dramatic peak referral times. Clerking efficiency was increased across all levels, with increased educational opportunities, including senior review. The medical registrar had greater oversight of the clerking force and ability to manage and anticipate work pressures. Senior review or post-take ward round (PTWR) was more timely. As a result, a greater number of ward admissions were achieved in-hours.

Working relationships between medicine and the ED were also enhanced. Flow through the ED was improved as a result of all the above, in particular, due to increased availability of real-time metrics and 'Decision to admit' (DTA) data. The application has identified causes of inappropriate referrals to medicine to tackle prior to consideration of an 'open' list. As a result of all the above, earlier specialist input was achieved; data has not yet been evaluated, however it is inferred that earlier specialist input results in reduced cost and overall length of stay.

\section{Conclusion}

The electronic workflow tool has enhanced performance and flow, both objectively and subjectively, improving workflow relations between the ED and medicine. Site management was also improved by ability to better prioritise and coordinate resources on the front line. This tool has demonstrated the need for greater transparency and real-time information to streamline process, prioritise, coordinate and root out delays, driving continuous improvement in care and flow. Further exciting applications expanding on this are being explored to enhance work flow and care generation.

Authors: ${ }^{\mathrm{A}}$ Croydon University Hospital; ${ }^{\mathrm{B}}$ Patienteer

${ }^{*} \mathrm{RCP}$ chief registrar 